\title{
Noise sources from a cylindrical cavity
}

\author{
M. Grottadaurea* and A. Rona ${ }^{\dagger}$ \\ University of Leicester, Leicester LE1 7RH, United Kingdom
}

\begin{abstract}
The expansion of air traffic operations is nowadays limited by environmental constraints on noise. Advances in jet noise reduction have increased the importance of landing noise from the airframe as a significant contribution to the effective perceived noise level around airports. The most acoustically active airframe components in a civil aircraft are the high lift systems and the landing gear. Nonetheless, other components, such as fuel vents or ailerons, also contribute to the overall noise emissions. This study considers a cylindrical cavity as a low fidelity fuel vent model. Different diameter to depth ratios and inflow velocities are simulated by means of an in-house compressible Euler solver. The predictions for a diameter to depth ratio of 0.714 show an unsteady asymmetric vortex structure at the inflow Mach numbers of 0.235 and 0.3. The alternate impingement of this vortex on the right and on the left of the cavity trailing edge produces pressure waves and the flow instability is self-sustained. The simulations of a cavity with a length to depth ratio of 2.5 at the same Mach numbers show a similar self-sustained instability where the flow recirculation is symmetric about the cavity mid-plane. To identify and localize the most acoustically active regions in the inviscid flow model, the double divergence of the Lighthill stress tensor was computed from the aerodynamic predictions. This work sets the basis to perform a Ffowcs Williams and Hawkings acoustic analogy to predict the fuel vent contribution to landing noise.
\end{abstract}

\section{Nomenclature}

$c \quad$ Speed of sound

$\delta(f)$ Dirac delta function

e Internal energy

$\mathbf{F} \quad$ Inviscid fluxes, $\mathbf{F}=[\rho \mathbf{u}, \rho \mathbf{u} \otimes \mathbf{u}+p \mathbb{I}, \rho \mathbf{u}(e+p / \rho)]^{\mathrm{T}}$

$f \quad$ Characteristic frequency

$f=0$ Function describing the integration surface

$\nabla \quad$ Gradient operator

$H(f)$ Heaviside function, $H(f)=0$ for $f<0$ and $H(f)=1$ for $f>0$

II Identity matrix

$M \quad$ Mach number

n Unit outward normal vector to $f=0$

$p \quad$ Pressure

$p^{\prime} \quad$ Acoustic pressure fluctuation, $p^{\prime}=p-p_{\infty}$

$r \quad$ Distance between observer and sources, $r=\|\mathbf{x}-\mathbf{y}\|$

$\rho \quad$ Density

$\rho^{\prime} \quad$ Acoustic density fluctuation

SPL Sound Pressure Level

St Stroual number $S t=f L / u_{\infty}$

$s \quad$ Entropy

$T$ Temperature

T Lighthill stress tensor with component $T_{i, j}=\rho u_{i} u_{j}+\left(p^{\prime}-c^{2} \rho^{\prime}\right) \delta_{i, j}$

$\mathbf{U} \quad$ Conservative variable vector, $\mathbf{U}=[\rho, \rho \mathbf{u}, \rho e]^{\mathrm{T}}$

${ }^{*}$ PhD Student, Department of Engineering.

${ }^{\dagger}$ Lecturer, Department of Engineering and AIAA Member. 


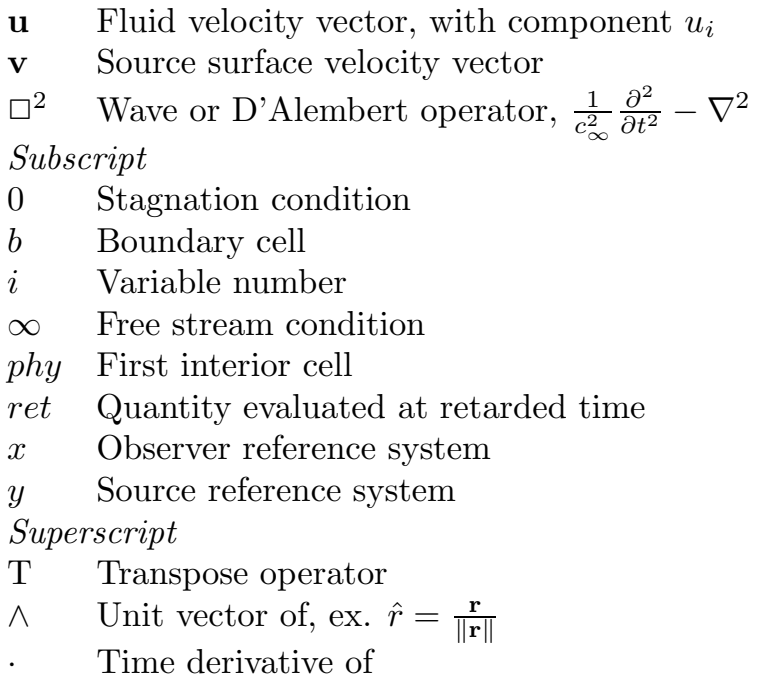

\section{Introduction}

Recent advances in jet noise reduction have considerably increased the importance of noise from the engine fan and the airframe as a significant contribution to the overall noise, especially during landing. Civil airframes often feature recesses or grooves to accommodate service hatches and other ancillary equipment. The flow in these cavity-shaped recesses is unsteady and, at typical landing speeds, may features large-scale instabilities. The most acoustically active airframe components in a civil aircraft are the high lift systems and the landing gear. Nonetheless, other components, such as fuel vents or ailerons, also contribute to the overall noise emissions. A cylindrical open cavity placed one metre downstream of the wing leading edge is herein investigated as an initial low fidelity fuel vent model of a widebody civil aircraft. The fuel vent location and design can vary among aircraft. Fuel vents are often cut in the underside wing skin or are located on the wing trailing edge. They avoid any vacuum in the fuel tank that could stop the fuel flow or cause the tank to implode, by letting the air in to balance the tank internal pressure. Although the amplitude of fuel vent noise is relatively low compared to other airframe noise sources, it happens to cover a higher frequency range, therefore it is perceived by a ground observer as louder with respect to what its amplitude in decibel would suggest, due to the $\mathrm{dB}(\mathrm{A})$ weighting. ${ }^{1}$

Recent advances in code parallelization and in multi-processor computational platforms allow nowadays the modelling of representative industrial geometries by conventional Computational Fluid Dynamics (CFD). ${ }^{2}$ Different challenges are nonetheless posed by computational aeroacoustics. A larger computational domain with respect to conventional CFD is required to resolve a full wavelength of the radiated noise. A more accurate, less dissipative and efficient non-reflective boundary condition needs to be applied to correctly evaluate the amplitude of the pressure fluctuations in the near-field acoustic domain. High-order low dissipation and dispersion schemes have lowered the cost of aeroacoustic models to a more affordable level.

Direct numerical simulation (DNS) of the radiating noise is limited to low Reynolds number and high Mach number models. To resolve all the relevant scales of motion in the flow and to cover at least one wavelength of the radiated sound in the computational domain, the total number of necessary operations is of the order of the Reynolds number to the third power over the Mach number to the fourth power, for a fully three dimensional simulation. ${ }^{3}$ DNS is more suitable for simpler aeroacoustic models, specifically to validate an aeroacoustic method that includes a turbulence closure model. Past cavity aeroacoustic investigations mainly focussed on rectangular enclosures, due to the savings in computer time that can be achieved by the use of a Cartesian mesh. The computational resources now available enable the use of a curvilinear conformal mesh to tackle also a cylindrical cavity. ${ }^{4,5}$

To obtain a preliminary understanding of the main instability mechanism and of the overall flow pattern that characterizes the cavity flow, an inviscid simulation is herein used. This simulation is expected to capture the main cavity flow instability mechanism. However, this method omits to account for the inflow turbulent boundary layer. From the available literature, ${ }^{6}$ it is known that the momentum thickness $(\theta)$ at the leading edge of the cavity plays an important role in the selection of the modes and in governing the 
growth of the shear layer ${ }^{6,7,8}$ spanning an open cavity. ${ }^{9}$ At the selected flow regime, the interaction of the shear layer with the downstream cavity edge produces pressure waves that excite the leading edge shear layer, providing the feedback for self-sustained oscillations. The evolution of such unsteady pressure field and its interaction with the leading edge shear layer can be obtained by modelling the inflow boundary layer as a vortex sheet, neglecting the inflow momentum thickness. ${ }^{10}$ Therefore the Euler model herein considered can approximately capture this feedback, responsible for the tonal component of the cavity noise. Though the spectrum of cavity noise typically contains broadband and tonal components, and the broadband components are introduced by the turbulence in the shear-layer spanning the cavity opening, cavity tones often dominate the far-field noise spectrum. The frequencies of such tones can be approximately reproduced by an Euler method.

\section{Test-case geometry and inflow condition}

Two cylindrical cavities of diameter (L) of $100 \mathrm{~mm}$ and depths (D) of $140 \mathrm{~mm}$ and $70 \mathrm{~mm}$ are herein studied as low fidelity fuel vent models. The characteristic length and width are equal to the diameter, which leads to a flow with important spanwise structures. ${ }^{11}$ Upstream of the cavity, the inflow is uniform. Two different free-stream velocities are considered in this study $u_{\infty}=102 \mathrm{~m} / \mathrm{s}$ and $u_{\infty}=80 \mathrm{~m} / \mathrm{s}$ at International Standard Atmospheric ground conditions. At these conditions, the resulting non-dimensional parameter that characterizes the numerical predictions are: $L / D=0.714, L / D=2.5, M_{\infty}=0.235$, and $M_{\infty}=0.3$.

\section{Numerical method}

\section{III.A. Governing equations}

The conservative time-dependent Euler equations are:

$$
\frac{\partial}{\partial t} \mathbf{U}+\nabla \cdot \mathbf{F}=0
$$

Taking the time derivative of the conservation of mass and the divergence of the conservation of momentum, Lighthill ${ }^{12}$ rearranged equation (1) into an inhomogeneous wave equation where the sources of noise are on the right hand side:

$$
\square_{y}^{2} p^{\prime}(\mathbf{y}, t)=\nabla_{y} \cdot \nabla_{y} \cdot \mathbf{T}
$$

\section{III.B. Discretization and integration methods}

To compute the conservative variables $\mathbf{U}$ in the computational domain, an in-house solver is used. The flow solver is a finite volume approximate Riemann solver with a Monotone Upstream-centered Scheme for Conservation Laws (MUSCL) interpolation. ${ }^{13}$ The latter, by Van Leer et al., ${ }^{14}$ gives up to a third order accurate reconstruction of the spatial gradient in region of smooth flow. To prevent numerical oscillations in region of rapidly changing flow, the spatial gradients are limited by the introduction of a Total Variation Diminishing Scheme (TDV) by Sweby. ${ }^{15}$ The scheme is explicit and a standard multi-stage Runge-Kutta (RK) integration is used to time-march the flow. A second order two step RK is used in the simulation.

\section{III.C. Boundary conditions}

The computational domain is fully rinded using ghost cells, generated in the code by mirroring the first interior cell about the boundary plane. Boundary flow states are imposed in the ghost cell. In the Euler simulation, an inviscid wall condition, or slip condition, is imposed in the physical wall boundaries and a subsonic non-reflective far-field boundary condition in the domain outer boundaries. The latter is a switching condition between a subsonic inflow condition and a subsonic outflow condition, depending on the value of the inner flow normal velocity component with respect to the surface boundary, according to CFD General Notation System standard. ${ }^{16}$

The inviscid wall condition corresponds to $\mathbf{u} \cdot \mathbf{n}=\mathbf{0}$ at the boundary $S$ between the most interior cell and the ghost cell, where $\mathbf{n}$ is the inward normal to $S$. This is numerically achieved by imposing:

$$
\mathbf{u}_{b}=\mathbf{u}_{p h y}-2 \mathbf{u}_{p h y} \cdot \mathbf{n}
$$


To obtain a non-reflective boundary condition, a $3 \mathrm{D}$ extension ${ }^{17}$ of the characteristic based boundary conditions of Giles $^{18}$ is used. To limit the non-physical reflection caused by the numerical scheme, the following procedure is followed, depending on whether the flow is entering or exiting the computational domain boundary. In the far-field, outside the computational domain, the following conditions are known: $\mathbf{u}_{\infty}, p_{\infty}$ and $T_{\infty}$. Based on these value, $c_{\infty}, s_{\infty}, T_{0}, p_{0}$ and $\rho_{0}$ are evaluated. Using $\mathbf{U}_{b}$ is possible to define $c_{b}$ and $s_{b}$. An average speed of sound $c=\left(c_{\infty}+c_{b}\right) / 2$ is then derived. Based on average speed of sound, $\rho, \mathbf{u}$ and $p$ are computed using $s_{b}$ and $\mathbf{u}_{b}$ if the flow is entering the domain, and $s_{\infty}$ and $\mathbf{u}_{\infty}$ if the flow is exiting the domain.

\section{III.D. Acoustic sources}

Equation (2) describes the problem of the non-linear propagation of the acoustic waves in the flow reference system. For many applications, an absolute reference system is more suitable for studying the radiated noise from the moving sources. Following the work of Curle, ${ }^{19}$ Ffowcs Williams and Hawkings ${ }^{20}$ derived equation (1) in an absolute reference system including the effect of surfaces in arbitrary motion. In the absolute reference system, the conservation of mass can be written as: ${ }^{21}$

$$
\frac{\partial}{\partial t} \rho+\nabla_{x} \cdot \rho \mathbf{u}=\left(\rho_{\infty} \mathbf{u} \cdot \mathbf{n}\right) \delta(f)+\left(\rho-\rho_{\infty}\right)(\mathbf{u} \cdot \mathbf{n}-\mathbf{v} \cdot \mathbf{n}) \delta(f)
$$

The conservation of momentum can be written as: ${ }^{21}$

$$
\frac{\partial}{\partial t}(\rho \mathbf{u})+\nabla_{x} \cdot(p \mathbb{I}+\rho \mathbf{u} \otimes \mathbf{u})=\left[\left(p-p_{\infty}\right) \mathbb{I} \cdot \mathbf{n}\right] \delta(f)+[(\rho \mathbf{u})(\mathbf{u} \cdot \mathbf{n}-\mathbf{v} \cdot \mathbf{n})] \delta(f)
$$

Following the same procedure in section III.A, the Ffowcs Williams and Hawkings acoustic analogy $(\mathrm{FW}-\mathrm{H})$ is obtained subtracting the divergence of equation (5) from the time derivative of equation (4): ${ }^{21,22}$

$$
\begin{aligned}
\square_{x}^{2} p^{\prime}(\mathbf{x}, t)= & \nabla_{x} \cdot \nabla_{x} \cdot[\mathrm{T} H(f)] \\
& -\nabla_{x} \cdot\{[p \mathbb{I} \cdot \mathbf{n}+\rho \mathbf{u}(\mathbf{u} \cdot \mathbf{n}-\mathbf{v} \cdot \mathbf{n})] \delta(f)\}+\frac{\partial}{\partial t}\left\{\left[\rho_{\infty} \mathbf{v} \cdot \mathbf{n}+\rho(\mathbf{u} \cdot \mathbf{n}-\mathbf{v} \cdot \mathbf{n})\right] \delta(f)\right\}
\end{aligned}
$$

It is of interest to notice that no assumption has been made in equation (6) on the integration surface $f=0$ that can be porous. Equation (6) can be integrated in retarded time using the Green function method. Farassat ${ }^{23}$ introduced the following relation to use the time resolved CFD data as input to the integral formulation of FW-H:

$$
\begin{gathered}
\frac{\partial}{\partial x_{i}}\left[\frac{\delta(f)}{r}\right]_{r e t}=-\frac{1}{c} \frac{\partial}{\partial t}\left[\frac{r_{i} \delta(f)}{r^{2}}\right]_{r e t}-\frac{r_{i} \delta(f)}{r^{3}} \\
\frac{\partial}{\partial t}[Q(\mathbf{y}, \tau)]_{r e t}=\left[\frac{1}{1-M_{r}} \frac{\partial Q}{\partial \tau}\right]_{r e t}
\end{gathered}
$$

In equation (8), $Q(\mathbf{y}, \tau)$ is a generic function in the source reference system $y$ and $M_{r}=\mathbf{u} \cdot \hat{\mathbf{r}} / c$ is the Mach number in the observer direction. Integrating equation (6) in retarded time and introducing equation (7) and (8) in the result gives: ${ }^{21,22}$

$$
\begin{aligned}
4 \pi p^{\prime}= & \int_{f=0}\left[\frac{\rho_{\infty}(\dot{\mathbf{u}} \cdot \mathbf{n}+\mathbf{u} \cdot \dot{\mathbf{n}})}{r\left(1-M_{r}\right)^{2}}\right]_{r e t} d S+\int_{f=0}\left[\frac{\rho_{\infty}(\mathbf{u} \cdot \mathbf{n} K)}{r^{2}\left(1-M_{r}\right)^{3}}\right]_{r e t} d S \\
& +\frac{1}{c} \int_{f=0}\left[\frac{\dot{\mathbf{F}} \cdot \hat{\mathbf{r}}}{r\left(1-M_{r}\right)^{2}}\right]_{r e t} d S+\int_{f=0}\left[\frac{\mathbf{F} \cdot \hat{\mathbf{r}}-\mathbf{F} \cdot \mathbf{u} / c}{r^{2}\left(1-M_{r}\right)^{2}}\right]_{r e t} d S \\
& +\frac{1}{c} \int_{f=0}\left[\frac{\mathbf{F} \cdot \hat{\mathbf{r}} K}{r^{2}\left(1-M_{r}\right)^{3}}\right]_{r e t} d S
\end{aligned}
$$

Where the quadrupole term contribution has been neglected. In equation (9), $K=\dot{M}_{r} r+M_{r} c-M_{r}^{2} c$ and $\mathbf{F}=\left[p^{\prime} \mathbb{I}+\rho \mathbf{u} \otimes(\mathbf{u}-\mathbf{v})\right] \cdot \mathbf{n}$. 


\section{Numerical model}

\section{IV.A. Computational mesh}

To model the cylindrical cavity of section II, a conformal curvilinear mesh is used. By multi-zone decomposition, the flow domain has been divided into six zones, as shown in figure 1. Each zone has a similar number of cells to even out the computational load among the processors.

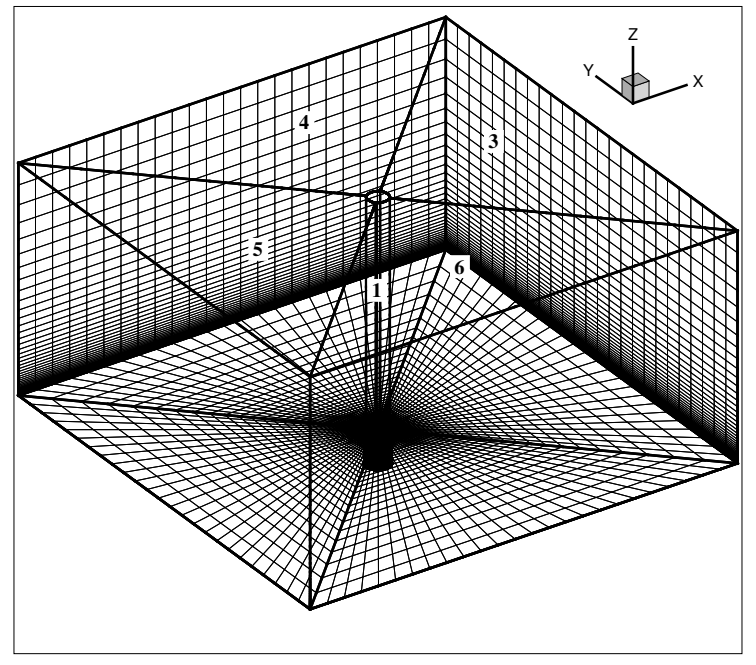

(a) Computational mesh

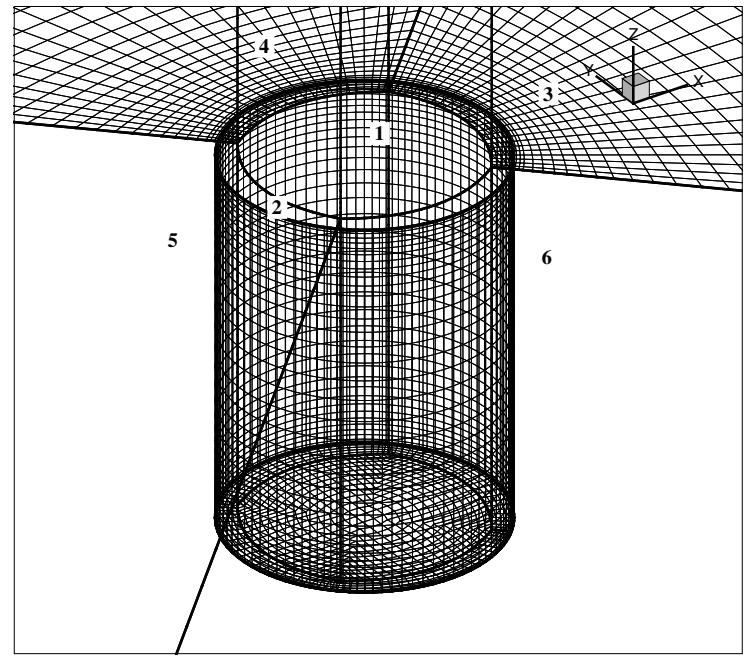

(b) Detail of the mesh in the enclosure

Figure 1. Computational domain details

The domain outside the cavity is $18.4 L \times 18.4 L \times 9.6 L$. A large domain of the order of $20 L$ is used to resolve at least one full acoustic wavelength of the radiating sound. The selected computational domain volume is the smallest that prevents the non-linear interaction of the acoustic radiation in the computational domain with the potential flow at the outer domain boundaries. ${ }^{6} 0.34$ million cells are used in the $L / D=0.71$ cavity model and 0.327 million cells in the $L / D=2.5$ model.

Figure 2 shows the mesh skewness ${ }^{24}$ over the cavity opening. The skewness has the maximum value of 0.42. As the skewness is an index of the local mesh deformation, it is best minimized throughout the domain. An advantage of the generated computational mesh is the modest deformation of the cells around the perimeter of the cylinder.

\section{IV.B. Boundary conditions and starting flow condition}

The following boundary conditions are used in the model: the physical walls are modelled as an inviscid wall, the outer domain boundaries are far-field and the inter-block boundaries are connectivity. An inter-block boundary cell is defined as the ghost cell corresponding to physical cells of a neighbouring zone. For a general connectivity between zone 1 and zone 2 , the state in the inter-block boundary ghost cells is defined as $U_{b 1}=U_{p h y 2}$. The face $x=9.2 L$ of zone 3 is defined as an outflow condition and the face $x=-9.2 L$ of zone 5 is defined as an inflow condition. The inflow and outflow conditions are obtained from the far-field condition in section III.C by fixing the flow direction. In the far-field, two flow states $\left(\mathbf{u}_{\infty}, p_{\infty}, T_{\infty}\right)$ are used for $M_{\infty}=0.235$ and $M_{\infty}=0.3$. These are $(102.1,0,0)^{T} \mathrm{~m} / \mathrm{s}, 101325 \mathrm{~Pa}, 298.15 \mathrm{~K}$ and $(80,0,0)^{T} \mathrm{~m} / \mathrm{s}$, $101325 \mathrm{~Pa}$ and $298.15 \mathrm{~K}$. At the inflow, $\mathbf{u}=\mathbf{u}_{\infty}$ and $\rho=\rho_{\infty}=1.225 \mathrm{~kg} / \mathrm{m}^{3}$ and at the outflow the back pressure $p=p_{\infty}$.

At the beginning of the simulation, the inflow is imposed over the full computational domain. The simulation converges to a statistically stationary oscillatory solution after $0.1 \sim 0.2$ million iterations a 0.22 the Courant number. 


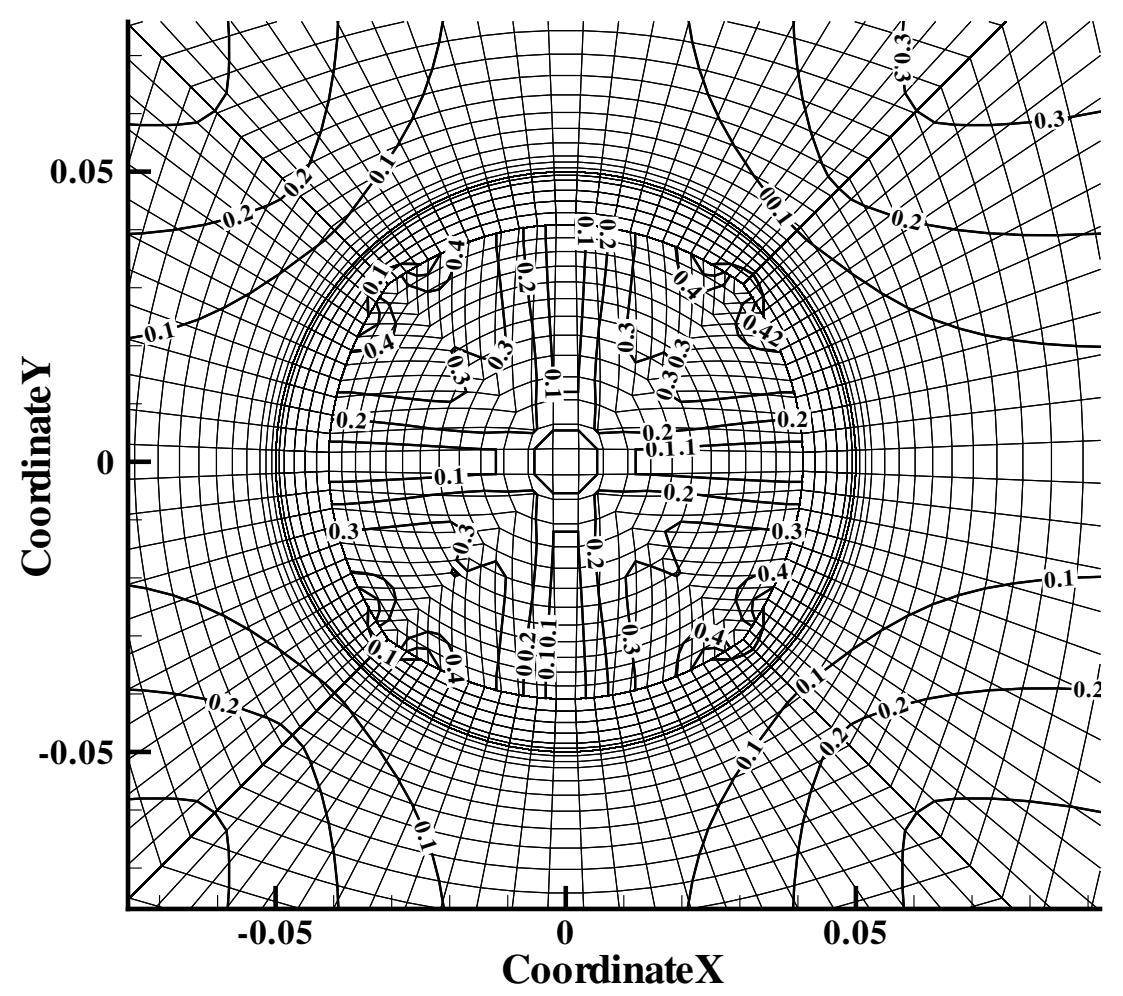

Figure 2. K plane skewness over the cavity open end 


\section{Unsteady aerodynamic predictions}

\section{V.A. Flow instability}

\section{V.A.1. Shallow cavity}

The $L / D=2.5$ shallow cavity configuration is characterized by a symmetric recirculation within the cavity at both $M_{\infty}=0.235$ and $M_{\infty}=0.3$, as shown in figures 3(a) and 3(b). The flow separates at the cavity leading edge forming a vortex sheet. The interaction between the fluctuating vortex sheet predicted by the Euler model and the cavity trailing edge produces pressure waves. ${ }^{25}$ The frequency that characterizes these pressure waves phase-locks the vortex sheet fluctuation, producing a self-sustained instability. The numerical predictions suggest that the recirculation pattern inside the enclosure is independent from the free-stream velocity but it is probably related to the cavity geometry. The $L / D=2.5$ cavity flow is found to have a dominant instability mode at $S t=0.833$ at $M_{\infty}=0.235$ and at $S t=1.448$ at $M_{\infty}=0.3$. The maximum reversed velocity inside the enclosure is approximately $u=-24 \mathrm{~m} / \mathrm{s}$ at $M_{\infty}=0.235$ and $u=-40 \mathrm{~m} / \mathrm{s}$ at $M_{\infty}=0.3$. The near-field pressure fluctuation peak directivity is $135^{\circ}$ from the $u_{\infty}$ direction.

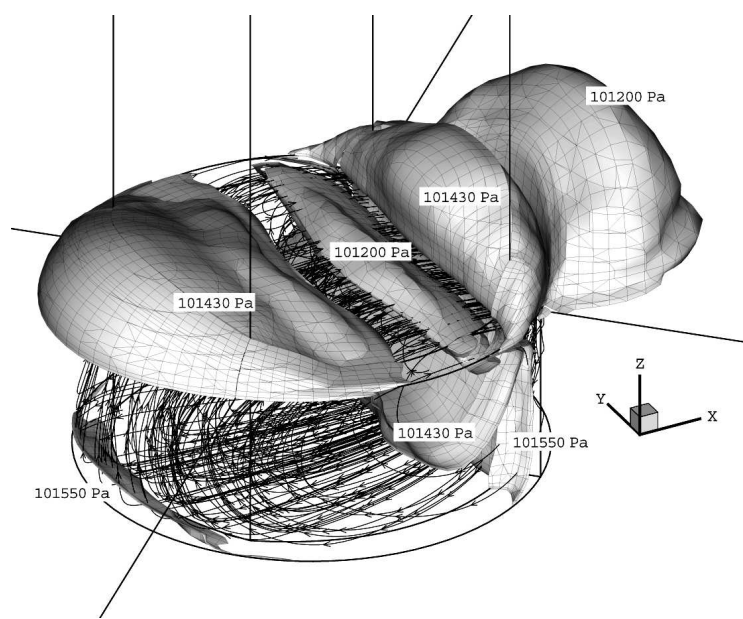

(a) Streamline and pressure iso-contour in the enclosure, $L / D=2.5$ and $M_{\infty}=0.235$

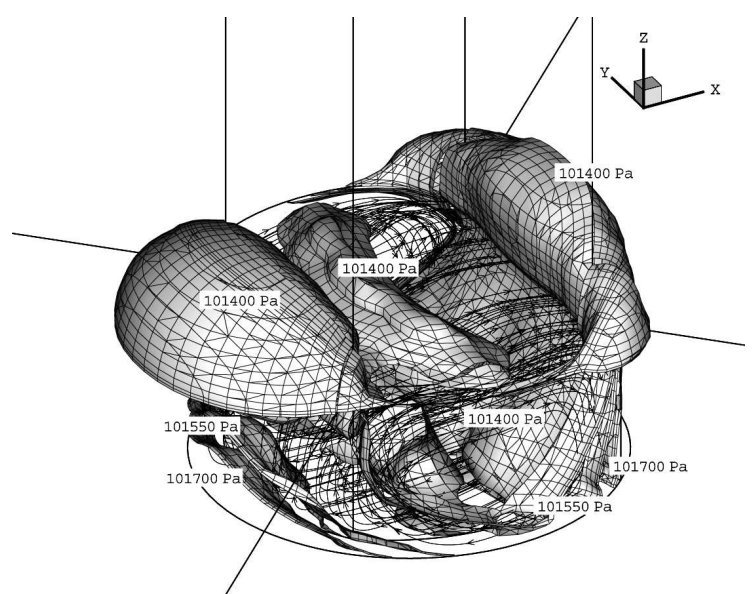

(b) Streamline and pressure iso-contour in the enclosure, $L / D=2.5$ and $M_{\infty}=0.3$

Figure 3. Symmetric recirculation from a $L / D=2.5$ shallow cavity

\section{V.A.2. Deep cavity}

The $L / D=0.71$ deep cavity configuration is characterized by an asymmetric recirculation in the enclosure, as shown in figure 4. A similar asymmetric recirculation in a $L / D=2$ cylindrical cavity was observed experimentally by Dybenko et al. ${ }^{5,26}$ The simulation suggests that the greater cavity depth may be the cause of the asymmetric recirculation and of the asymmetric pressure iso-contours in the cavity open end.

The $L / D=0.71$ cavity flow is found to have a dominant instability mode at $S t=0.5295$ at $M_{\infty}=0.235$ and at $S t=0.491$ at $M_{\infty}=0.3$. The maximum reversed velocity inside the enclosure is approximately $u=-50 \mathrm{~m} / \mathrm{s}$ at $M_{\infty}=0.235$ and $u=-63 \mathrm{~m} / \mathrm{s}$ at $M_{\infty}=0.3$.

\section{V.B. Near-field Sound Pressure Level}

\section{V.B.1. Shallow cavity}

The near-field SPL from a shallow cavity configuration is characterized by a low amplitude pressure fluctuation as shown in figure 5. $S P L=87 \mathrm{~dB}$ is predicted at $\mathbf{y}=(0,0,8 L)$ at $M_{\infty}=0.3$ and $S P L=86 \mathrm{~dB}$ is predicted at $\mathbf{y}=(0,0,8 L)$ at $M_{\infty}=0.235$. Comparing figure $5(\mathrm{a})$ and figure $5(\mathrm{~b})$, the near-field SPL iso-contours have a different shape, possibly due to the different Doppler effect that changes the orientation of the radiation peak directivity at $M_{\infty}=0.235$ with respect to $M_{\infty}=0.3$. 


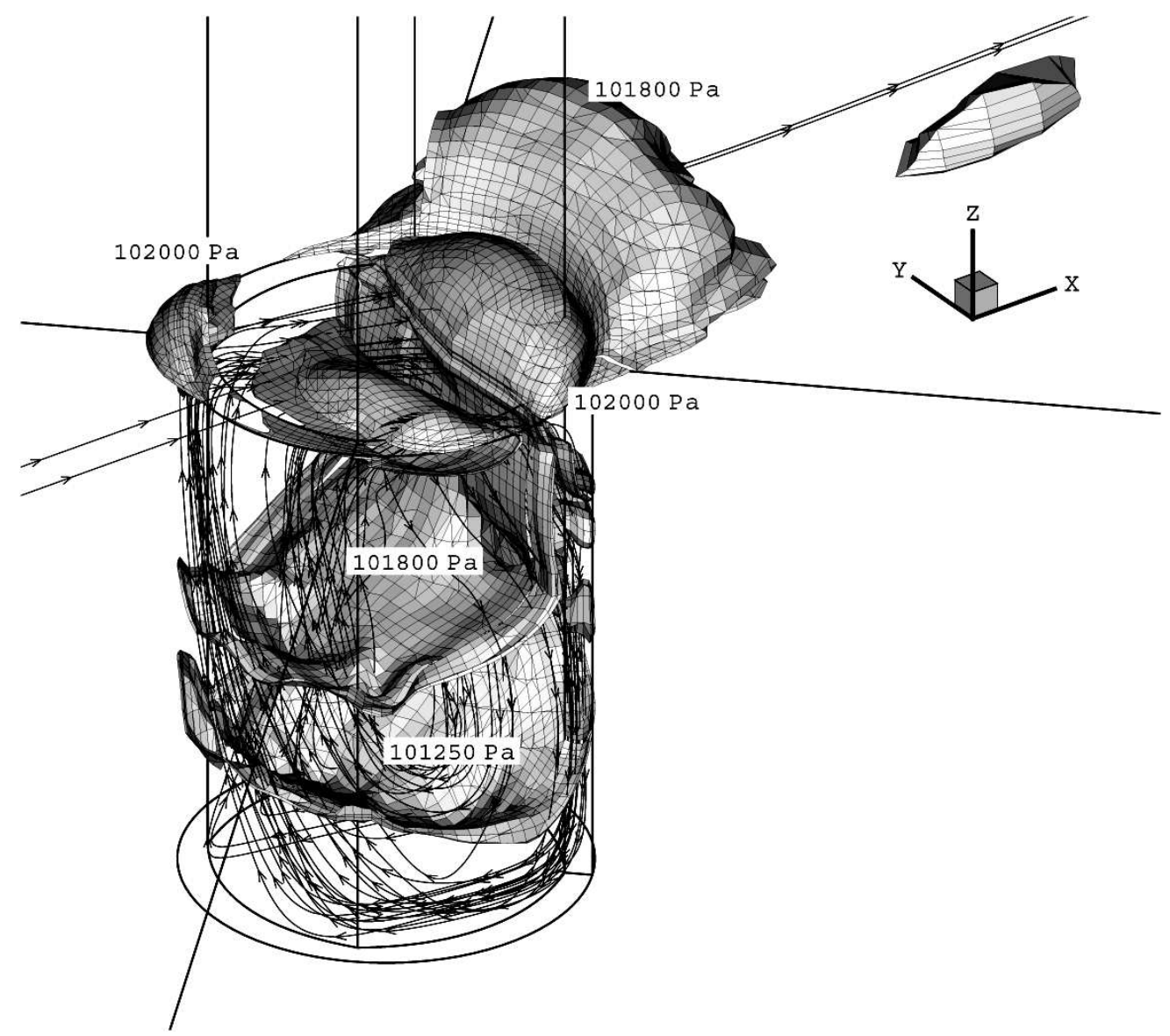

Figure 4. Asymmetric recirculation from a deep cavity configuration. Streamline and pressure iso-contour in the enclosure $L / D=0.71$ and $M_{\infty}=0.235$

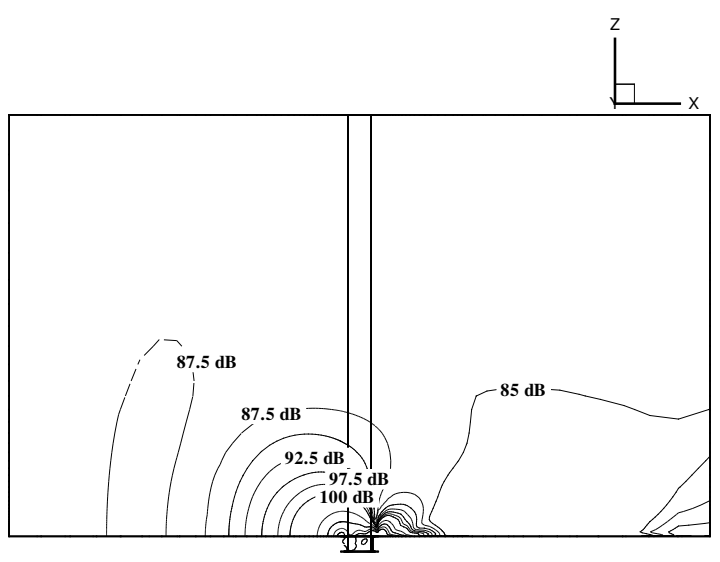

(a) Near-field SPL iso-contour line. $y=0$ plane, $M_{\infty}=0.235$

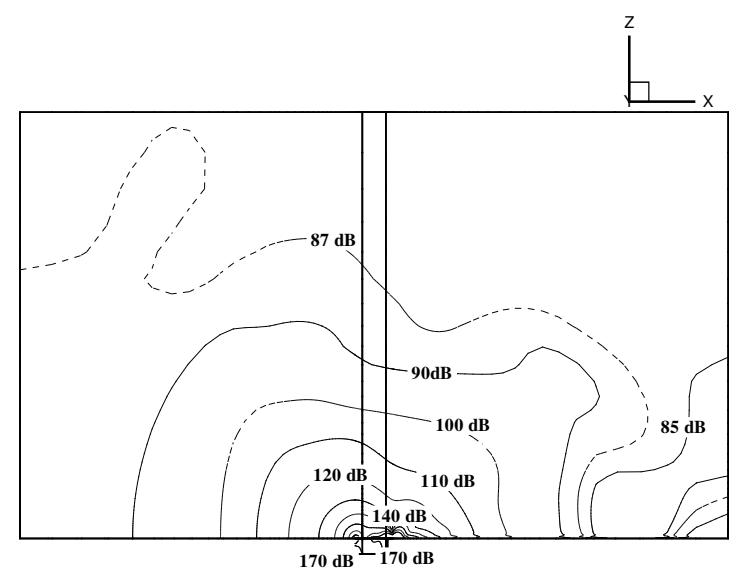

(b) Near-field SPL iso-contour line. $y=0$ plane, $M_{\infty}=0.3$

Figure 5. Near-field SPL from a $L / D=2.5$ deep cavity configuration 


\section{V.B.2. Deep cavity}

The near-field SPL has been evaluated using 25 frames over one period of oscillation. Comparing figure 6(a) and figure 6(b), the SPL iso-contours in acoustic near-field are similar. The amplitude of the pressure fluctuation decreases towards the domain boundaries without appreciable reflection, showing the good performance of the selected non-reflective boundary conditions. The SPL maxima are higher compared to those from the

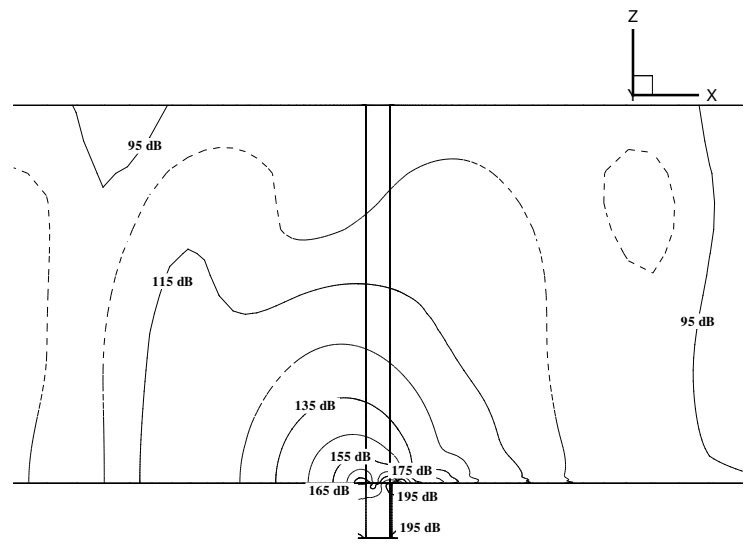

(a) Near-field SPL iso-contour line. $y=0$ plane, $M_{\infty}=0.235$

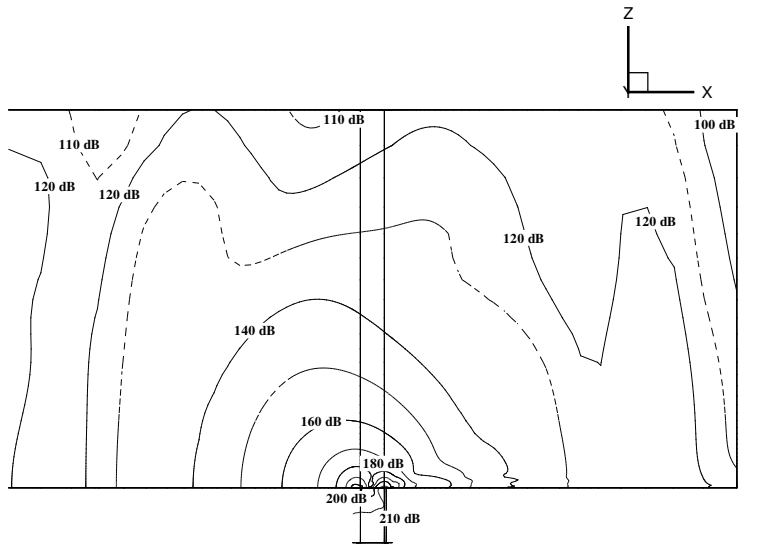

(b) Near-field SPL iso-contour line. $y=0$ plane, $M_{\infty}=0.3$

Figure 6. Near-field SPL from a $L / D=0.71$ deep cavity configuration

shallow cavity, suggesting that the azimuthal instability enhances the amplitude of the pressure fluctuations.

The numerical model predicts that the mass ejection occurs only on one side of the cavity trailing edge with respect to the streamwise direction, as shown in figure 4 . The higher pressure peak, characterizing this instability, could be related to the more localized interaction between the vortex sheet and the the cavity trailing edge side.

\section{V.C. Noise sources from the computational domain}

\section{V.C.1. Shallow cavity}

To identify the noise sources and to locate the integration surface of the FW-H acoustic analogy, the double divergence of the Lighthill stress tensor $\left(\nabla_{y} \cdot \nabla_{y} \cdot T\right)$ has been evaluated from the aerodynamic flow predictions. To differentiate $T_{i j}$, the altering equation toolbox from Tecplot 360 was used. The spatial derivative is only first order accurate and it does not perform well in the inter-block boundary cells, as shown in figure 7 . In the shallow cavity configuration, it is found that $\nabla_{y} \cdot \nabla_{y} \cdot T$ iso-contours, at $z=4 L$ plane above the cavity open end, coincide with those from pressure. The numerical result suggests that above this plane, the non-linear interaction related to the quadrupole term distribution can be neglected and the residual source field above this plane simply accounts for the Doppler effect of the free-stream. The appropriateness of the integration surface location can be checked by comparing the far field noise predictions from the $\mathrm{FW}-\mathrm{H}$ integral to the ones obtained from an integration surface moved further away from the enclosure.

\section{V.C.2. Deep cavity}

In the deep cavity configuration, where the instability is characterized by a lower $S t$ as described in V.A.2, a good agreement between the $p$ iso-contours and the $\left(\nabla_{y} \cdot \nabla_{y} \cdot T\right)$ iso-contours, shown in figure 8 , has been found at $z=8 L$ plane above the cavity open end. The large computational domain still allows different integration surfaces to be considered in the future work to compute the fuel vent contribution to the far-field noise. 


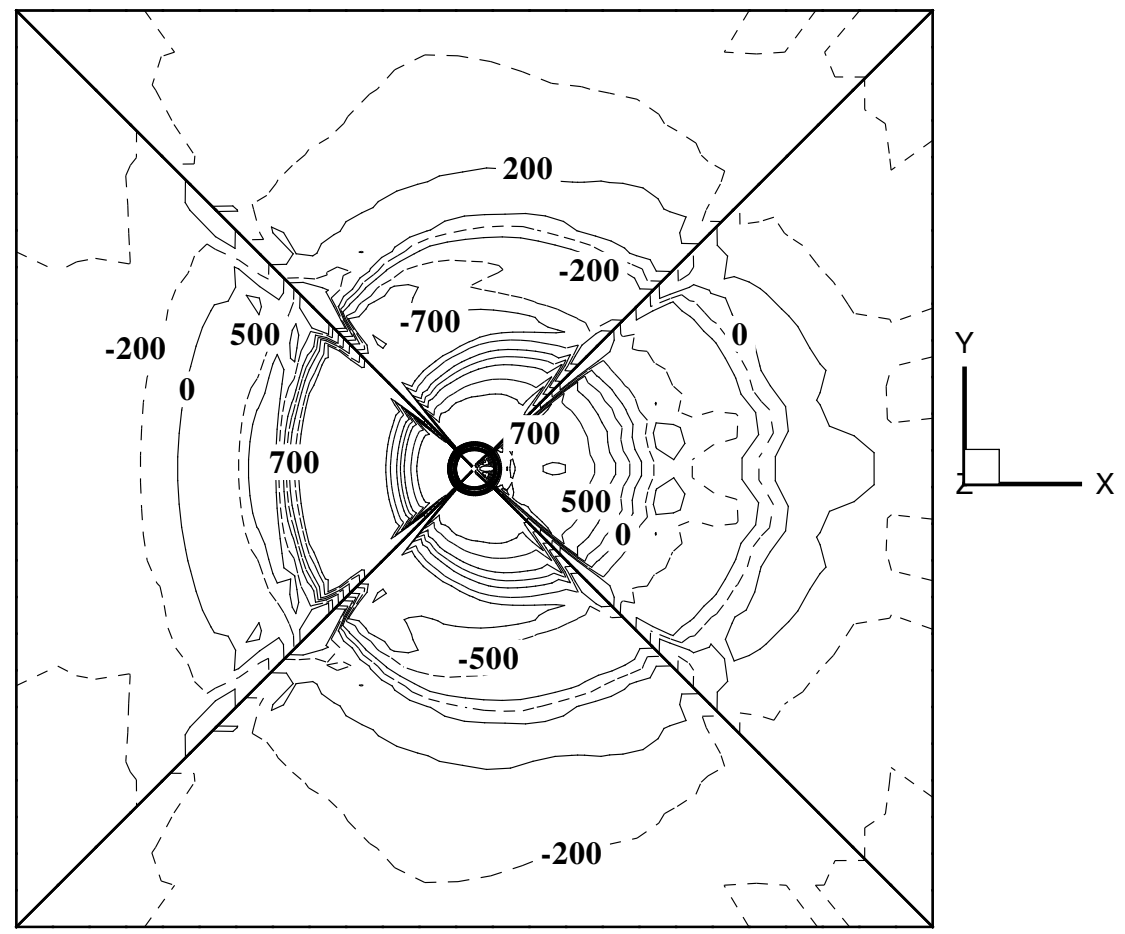

Figure 7. $\nabla_{y} \cdot \nabla_{y} \cdot T N / \mathrm{m}^{4} \cdot z=4 L L / D=2.5$ and $M_{\infty}=0.235[\mathrm{~m} / \mathrm{s}]$

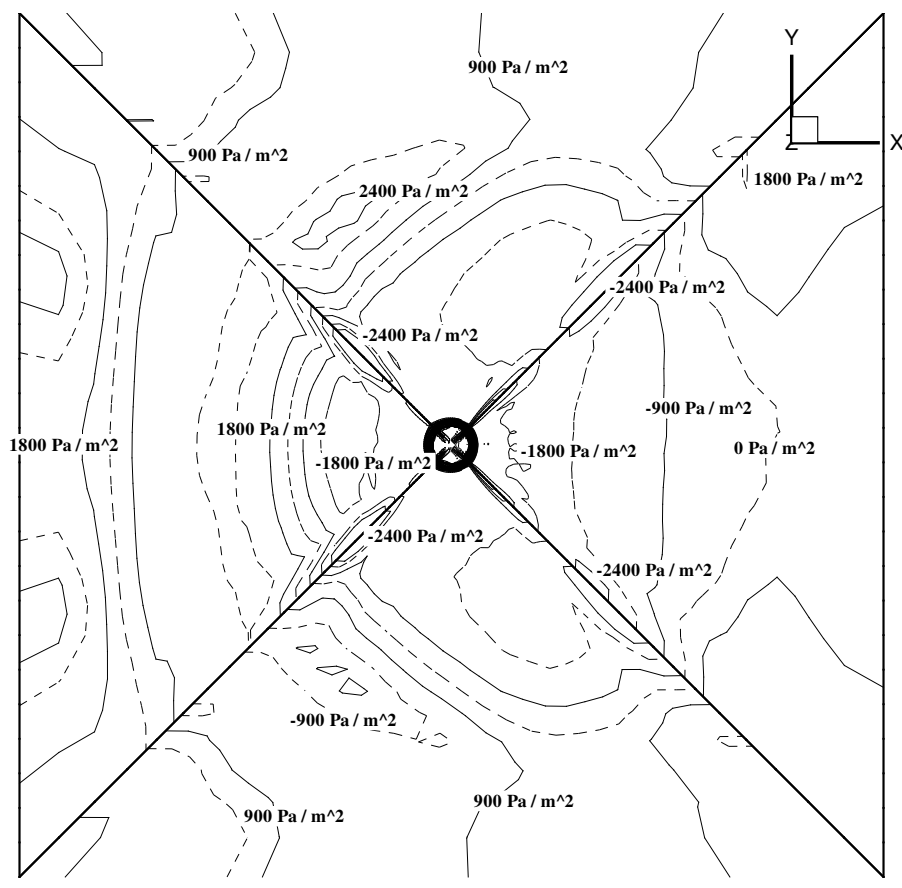

Figure 8. $\nabla_{y} \cdot \nabla_{y} \cdot T N / m^{4} \cdot z=8 L L / D=0.71$ and $M_{\infty}=0.235$ 


\section{Conclusion}

An Euler numerical model of a cylindrical cavity flow has been studied by an in-house CFD code. A $L / D=2.5$ shallow cavity has been modelled at two different free-stream condition $M_{\infty}=0.235$ and $M_{\infty}=0.3$. At the same free-stream conditions, a $L / D=0.71$ deep cavity was also modelled. The cavities are behaving as a closed cavity ${ }^{11}$ at the selected flow regimes and the geometries. The deep cavity configuration is characterized by an azimuthal instability, and by a recirculation at $45^{\circ}$ with respect to the streamwise direction. Mass ejection and injection occur only on one side of the cavity in this instability. The shallow cavity is characterized by a symmetric recirculation and by a lower amplitude pressure fluctuation. Although the pressure amplitude is lower in the shallow cavity, it happens to be at a higher frequency range. The SPL near-field has been estimated by averaging 25 frames over a period of oscillation. The numerical prediction gives an appreciation of the radiating noise characteristic directivity in the acoustic near-field. The double divergence of the Lighthill stress tensor has been valuated in the computational domain. The first order accurate results can be used to obtain a fist estimate for the location of the integration surface to perform a FW-H acoustic analogy.

\section{Acknowledgments}

This research project has been supported by a Marie Curie Early Stage Research Training Fellowship of the European Community's Sixth Framework Programme under contract number MEST CT 2005020301.

\section{References} 2006.

${ }^{1}$ Cambiano, M., Grottadaurea, M., and Rona, A., "Towards quieter airframes," Aeronautics Days, Vienna, Austria, July

${ }^{2}$ Long, L., Morris, P., and Agarwal, A., "A Review of Parallel Computing in Computational Aeroacoustics," Int. J. Computational Fluid Dynamics, Vol. 18, No. 6, 2004, pp. 493-502.

${ }^{3}$ Crighton, D. G., "Boundary conditions for direct computation of aerodynamic sound generation," Progress in Aerospace Sciences, Vol. 6, No. 1, 1975, pp. 31-96.

${ }^{4}$ Bunyakin, A. V., "Laminar boundary layer in a flow past an aerofoil with a circular cavity," Fluid Dynamics, Vol. 33, No. 2, 1998, pp. 196-200.

${ }^{5}$ Hering, T., Dybenko, J., and Savory, E., "Experimental verification of CFD modeling of turbulent flow over circular cavities," Canadian Society of Mechanical Engineering Forum, Kananaskis, Canada, May 2006.

${ }^{6}$ Colonius, T. and Lele, S., "Computational aeroacoustics: progress on nonlinear problems of sound generation," Progress on Aerospace Sciences, Vol. 40, 2004, pp. 365-416.

${ }^{7}$ Rowley, C., Colonius, T., and Basu, A., "On Self-sustained Oscillations in Two-dimensional Compressible Flow Over Rectangular Cavities," J. Fluid Mechanics, Vol. 455, 2002, pp. 315-346.

${ }^{8}$ Tam, C., "Computational Aeroacoustics: an Overview of Computational Challenges and Applications," Int. J. Computational Fluid Dynamics, Vol. 18, No. 6, 2004, pp. 547-67.

${ }^{9}$ Charwat, A., Roos, J., Dewey, F., and Hitz, J., "An investigation of separated flows. Part I: The pressure field," J. Aerospace Sci., Vol. 28, No. 6, 1961, pp. 457-70.

${ }^{10}$ Tam, C. and Block, P., "On the tones and pressure oscillations induced by flow over rectangular cavities," J. Fluid Mechanics, Vol. 89, 1978, pp. 373-99.

${ }^{11}$ Block, P., "Noise response of cavities of varying dimensions at subsonic speeds," TN D-8351, NASA, 1976.

${ }^{12}$ Lighthill, M., "Review of Computational Aeroacoustics Algorithms," Proceedings of the Royal Society of London. Series A (Mathematical and Physical Sciences), Vol. 267, 1961, pp. 147-82.

${ }^{13}$ Hirsch, C., Numerical Computation of INTERAL AND EXTERNAL FLOWS, Vol 2: Computational Methods for Inviscid Flows, Vol. 2, A Wiley-Interscience Publication, 1988.

${ }^{14}$ Van Leer, B., Thomas, J., Roe, P., and Newsome, R., "A comparison of numerical flux formulas for Euler and NavierStokes equation," AIAA 8th Computational Fluid Dynamics Conference, edited by AIAA, No. 87-1104, 1987, pp. 36-41.

${ }^{15}$ Sweby, P., "High resolution schemes using flux limiters for hyperbolic conservative laws," SIAM Journal of Numerical Analysis, Vol. 21, No. 5, October 1984, pp. 995-1011.

${ }^{16}$ AIAA, "Recommended Practice. The CFD General Notation System. Standard Interface Data Structures," AIAA R 101-2002, AIAA, Reston, VA, USA, 2002.

${ }^{17}$ Manna, M., A three dimensional high resolution compressible flow solver, Phd thesis, faculty of applied science, Université Catholique de Louvain, October 1992.

${ }^{18}$ Giles, M., "Non-reflecting boundary conditions for Euler Equation calculation," AIAA J., Vol. 28, No. 12, 1990, pp. 205058.

${ }^{19}$ Curle, N., "The Influence of Solid Boundaries upon Aerodynamic Sound," Proceedings of the Royal Society of London. Series A (Mathematical and Physical), Vol. 231, No. 1187, 1975, pp. 505-514.

${ }^{20}$ Ffowcs Williams, J. and Hawkings, D., "Sound generation by turbulence and surfaces in arbitrary motion," Proceedings of the Royal Society of London. Series A (Mathematical and Physical), Vol. 264, No. 1151, 1969, pp. 321-42. 
${ }^{21}$ Di Francescantonio, P., "A new boundary integral formulation for the prediction of the sound radiation," J. Sound and Vibration, Vol. 202, No. 4, 1997, pp. 491-509.

${ }^{22}$ Brentner, K. and Farassat, F., "An analytical comparison of the acoustic analogy and Kirchhoff formulation for moving surfaces," AIAA Journal, Vol. 36, No. 8, August 1998, pp. 1379-86.

${ }^{23}$ Farassat, F., "Linear acoustic formulas for calculation of rotating blade noise," AIAA Journal, Vol. 19, No. 9, March 1981, pp. 1122-30

${ }^{24}$ Grottadaurea, M. and Rona, A., "The role of the inflow momentum thickness in subsonic cylindrical cavity noise generation," 14th International Congress on Sound and Vibration, 2007.

${ }^{25}$ Powell, A., "Theory of vortex sound," J. Acoustical Society of America, Vol. 36, No. 1, 1964, pp. 177-95.

${ }^{26}$ Dybenko, J., Hering, T., and Savory, E., "Turbulent flow over circular cylindrical cavities with varying depth to diameter ratio," International Council of the Aeronautical Sciences Congress, Hamburg, Germany, September 2006. 